Original Article

Journal of Epilepsy Research pISSN 2233-6249 / eISSN 2233-6257

\title{
Spectrum of Cutaneous Adverse Reactions to Levetiracetam and Human Leukocyte Antigen Typing in North-Indian Patients
}

\author{
Bhargavi Ramanujam1, Kavish Ihtisham', Gurvinder Kaur², Shivani Srivastava1, \\ Narinder Kumar Mehra ${ }^{2}$, Neena Khanna ${ }^{3}$, Mahip Singh ${ }^{1}$, Manjari Tripathi ${ }^{1}$ \\ Departments of ${ }^{1}$ Neurology, ${ }^{2}$ Transplant Immunology and Immunogenetics, and ${ }^{3}$ Dermatology and Venereology, All \\ India Institute of Medical Sciences, New Delhi, India
}

\begin{abstract}
Background and Purpose: Aromatic antiepileptic drugs are frequently implicated for cutaneous adverse drug reactions (cADRs); there are case-reports of even severe reactions like drug reaction eosinophilia and systemic symptoms (DRESS) and Stevens Johnson syndrome (SJS)-toxic epidermal necrolysis with Levetiracetam (LEV). Certain human leukocyte antigen (HLA)-alleles have strong association with cADRs due to specific drugs - HLA-B*15:02 and HLA-A*31:01 in Carbamazepine (CBZ)-related SJS in Han-Chinese and European populations, respectively. Here, the spectrum of CADRs to LEV was studied, and HLA-typing in patients with cADRs due to LEV and some who were LEV-tolerant was performed, in an attempt to find an association between HLA and such reactions.
\end{abstract}

Methods: 589 patients taking LEV were screened for skin reactions, and eight patients with LEV-related CADRs and 25 LEV-tolerant controls were recruited - all 33 of North Indian ethnicity, their HLA-A, B, DRB1 genotyping done. Statistical analysis was done to compare carrier-rates and allele-frequencies of HLA-alleles between cases and controls (and healthy population, where necessary) for alleles occurring more than two times in either group.

Results: Out of 589 patients on LEV screened, there were 8 cases of CADR: 5 with maculopapular exanthema (MPE), 2 of SJS, and 1 with DRESS. Although HLA-A*33:01 was seen to occur more in MPE cases as compared to tolerant controls, the difference was not statistically significant (odds ratio [OR] 6.00, 95\% confidence interval $[\mathrm{Cl}]$ 0.30-116.6; $p=0.31$ ). HLA $* 11: 01$ and 24:02 were found to occur more in LEV-tolerant controls than in cases $(\mathrm{OR} 0.23[95 \% \mathrm{Cl} 0.02-2.36, p=0.33]$ and $1.00[95 \% \mathrm{Cl} 0.09-11.02, p=1.00]$ respectively).

Received May 4, 2016

Accepted June 5, 2016

Corresponding author: Manjari Tripathi Department of Neurology, Neurosciences Centre, All India Institute of Medical Sciences, New Delhi, India Tel. +91-11-26594494/26588248 Fax. $+91-26588248 / 26588166$ E-mail; manjari.tripathi1@gmail.com
Conclusions: Cutaneous reactions to LEV are very unusual, and their association with HLA in North-Indian population was not statistically significant. (2016;6:89-94)

Key words: Levetiracetam, Maculo-papular exanthem, Stevens-Johnson syndrome, Toxic Epidermal Necrolysis, Human leukocyte antigen (HLA)

\section{Introduction}

Cutaneous adverse drug reactions (CADRs) are commonly associated with antiepileptic drugs (AEDs) particularly the aromatic Carbamazepine (CBZ), Phenytoin (PHT), Phenobarbitone (PB), and Lamotrigine (LTG). They range from minor maculopapular exanthem (MPE) to severe life-threatening reactions like Drug reaction eosinophilia and systemic symptoms (DRESS), Stevens Johnson Syndrome (SIS) and Toxic epidermal Necrolysis (TEN), and manifest within a few hours to weeks of the AED being initiated. In a study by Sharma et al, out of 500 patients with CADRs with all drugs, anticonvulsants - PHT, CBZ and PB were implicated in $41.6 \%$ of patients with maculopapular rashes and $43.8 \%$ of life-threatening reactions - TEN and SJS. ${ }^{1}$ Although non-aromatic AEDs like Topiramate (TPM) and LEV are thought to be safer, there are several reports of even severe CADRs like SJS, DRESS due to LEV. ${ }^{2-4}$ In 2011, the United States Food and Drug Administration (US-FDA) included serious CADRs as potential safety risk from LEV in both paediatric and adult population. ${ }^{5}$ 
Cutaneous reactions to certain drugs including anticonvulsants have shown to have strong association with specific human leukocyte antigen (HLA) alleles - HLA-B*15:02 with CBZ-related SJS/TEN in the Han-Chinese, HLA-A*31:01 in European people. ${ }^{6,7}$ CBZ has been also found to be the causative drug in many other populations. Therefore, it is now recommended that HLA screening for $H L A-B^{*} 15: 02$ be done in the susceptible ethnic groups prior to initiation of the potentially offending drugs. Most of the studies with HLA typing in CADRs due to AEDs have been in CBZ-induced cases or those related to PHT or LTG, probably because of their much more frequent occurrence. In our study, we screened patients on LEV and performed HLA-typing of North Indian patients with a skin reaction to it, in an attempt to find a possible HLA-association with LEV-induced CADR in this population.

\section{Methods}

\section{Patient enrolment}

Five hundred and eighty-nine consecutive patients of epilepsy on LEV, presenting to the Neurology Outpatient Clinic or Emergency Department of the All India Institute of Medical Sciences (AlIMS) hospital between February 2013 and March 2015 were screened. All those developing skin rash, mucosal ulcers or both, within three months of initiation of LEV were recruited. These patients were all from the North Indian states of Bihar, Uttar Pradesh, Delhi, Haryana or Punjab. Dermatologist's opinion was taken to confirm the cutaneous drug reaction and to classify the same. Twenty-five patients, also from one of the above-mentioned North-Indian states, who had no skin reactions to LEV even after six months of taking the drug were enrolled as controls. A written informed consent was taken from all subjects, and the study was approved by the Institutional Ethics Committee. History, general physical, neurological and dermatological examination findings were recorded in a proforma.

\section{HLA genotyping}

$5 \mathrm{~mL}$ blood sample was drawn from all recruited subjects; De-oxyribonucleic acid (DNA) was extracted and checked for quality and quantity on $0.8 \%$ Agarose gel and spectrophotometer. HLA-A, B and DRB1 allele typing was done by Luminex based SSO technology (Lab type SSO HLA-B, A and DRB1 kits).

\section{Statistical analysis}

Two-tailed Fisher's exact test was done to determine statistical significance of the difference in carrier-rates between cases of MPE and controls for alleles occurring more than two times in either group. Also, odds ratio (OR) and allele frequency (AF) - number of times the allele is present in the sample divided by twice sample-size, expressed as a percentage - were calculated for these alleles.

If the AF was higher among tolerant controls than cases, the two-tailed Fisher's exact test was done to find statistical significance in the difference of carrier-rates and AFs in the former group and in a healthy North Indian population.

\section{Results}

Out of the 589 patients on LEV, 107 were on monotherapy and 482 on polytherapy. In all, eight patients developed CADR to LEV and were recruited as cases; of these, five were on LEV monotherapy and

Table 1. Clinical characteristics of cases with LEV-induced CADR

\begin{tabular}{|c|c|c|c|c|c|c|}
\hline & $\begin{array}{l}\text { Age (years)/ } \\
\text { Gender }\end{array}$ & Diagnosis & Phenotype & $\begin{array}{c}\text { Dose at which CADR } \\
\text { occurred }\end{array}$ & $\begin{array}{l}\text { Latency to CADR } \\
\text { (days) }\end{array}$ & Concurrent Drug \\
\hline Case 1 & $38 / \mathrm{M}$ & LRE-Right parietal lobe NCC & MPE & 500 mg twice a day & 15 & Nil \\
\hline Case 2 & $45 / F$ & LRE-left frontal lobe NCC & MPE & 1,000 mg twice a day & 13 & Nil \\
\hline Case 3 & $14 / F$ & LRE-left frontal FCD & MPE & 250 mg twice a day & 7 & $\begin{array}{l}\text { CBZ taken without } \\
\text { CADR for 1year }\end{array}$ \\
\hline Case 4 & $18 / F$ & JME & SIS & 250 mg twice a day & 10 & Nil \\
\hline Case 5 & $24 / \mathrm{M}$ & JME & SIS & 500 mg twice a day & 7 days & Nil \\
\hline Case 6 & $22 / F$ & JME & DRESS & 750 mg twice a day & 15 days & OXC, LTG \\
\hline Case 7 & $24 / F$ & LRE-Post stroke & MPE & 500 mg twice a day & 15days & $\begin{array}{l}\text { Clopidogrel, } \\
\text { Losartan. }\end{array}$ \\
\hline Case 8 & $22 / F$ & Idiopathic generalized epilepsy & MPE & 1,000 mg twice a day & 5 days & Nil \\
\hline
\end{tabular}

LEV, levetiracetam; CADR, cutaneous adverse drug reaction; LRE, localization-related epilepsy; NCC, Neurocysticercosis; MPE, maculopapular exanthem; SJS, Stevens Johnson syndrome; FCD, focal cortical dysplasia; CBZ, Carbamazepine; JME, juvenile myoclonic epilepsy; DRESS, drug reaction eosinophilia and systemic symptoms; OXC, Oxcarbazepine; LTG, lamotrigine. 
three on polytherapy. Mean age of the cases was 25.23 (range 14-45 years), there were six females and two males; their clinical features are tabulated (Table 1). Of the 25 controls, 13 were female and 12 were male; mean age was 26.68 (range 13-54 years), and twelve were on LEV alone, rest were also on other AEDs. None of the recruited subjects had a history of CADR to any drug previously.

HLA-A*11:01, 24:02, 33:01, 33:02, 68:01, 26:01, 01:01, 02:01 HLA B*7:02, 35:01, 40:01, 40:02, 40:06 15:01, 44:02 and HLA $D R B 1 * 15: 01,15: 02$ 07:01, 13:01, 14:01, 10:01 were the alleles that occurred more than twice in the MPE cases and/or the control-groups; their AF and OR are presented in Table 2., ${ }^{8,9}$ When the Fisher exact test was applied to carrier rates and AFs between the groups, the differences were not statistically significant (Table 2), The $\mathrm{AF}^{\prime} \mathrm{s}$ of HLA-A*11:01 was higher in tolerant controls (26\% versus
$10 \%$ in MPE cases and $12.5 \%$ in healthy population), and HLA-A*33:01 was higher among MPE cases (20\% versus $2 \%$ and $0.05 \%$ in tolerant controls and healthy population respectively). HLA-DRB1*13:01, 07:01 and 15:01 were also found to be more in tolerant controls than MPE cases (OR 2.66 [95\% Cl 0.34-20.50, $p=$ 0.56]; OR 2.66 [95\% Cl 0.34-19.06, $p=0.36]$; OR $0.27[95 \% \mathrm{Cl}$ $0.02-2.77 p=0.62]$ ).

However, the differences in occurrence-rates of alleles between MPE cases and tolerant controls were not statistically significant. Notably, one case of SJS was positive for HLA-B*15:02 in our study, and had been on CBZ for over a year before that without any cutaneous reactions. The four digit alleles of all the 33 subjects have been tabulated (Table 3).

Table 2. Allele frequencies and odds ratios of alleles occurring more than twice in MPE cases and/or control-groups

\begin{tabular}{|c|c|c|c|c|c|c|}
\hline \multirow[b]{2}{*}{ Alleles } & \multicolumn{2}{|c|}{ Allele frequency (\%) } & \multirow{2}{*}{ Odds ratio } & \multirow{2}{*}{$95 \% \mathrm{Cl}$} & \multirow{2}{*}{$\frac{\text { Fisher exact test- } p \text {-values }}{\text { Cases vs. Controls }}$} & \multirow{2}{*}{$\begin{array}{l}\text { Allele frequency in normal } \\
\text { population (\%) }\end{array}$} \\
\hline & Cases & Controls & & & & \\
\hline HLA-A*11:01 & 10 & 26 & 0.23 & $0.02-2.36$ & 0.33 & $12.5^{*}$ \\
\hline HLA-A*33:01 & 20 & 2 & 6.00 & $0.30-116.6$ & 0.31 & $0.50^{\dagger}$ \\
\hline HLA-A*33:03 & 20 & 6 & 1.83 & $0.15-22.36$ & 0.53 & $8.65^{*}$ \\
\hline HLA-A*24:01 & 10 & 6 & 1.83 & $0.15-22.36$ & 0.53 & $11.1^{\dagger}$ \\
\hline HLA-A*24:02 & 10 & 10 & 1.00 & $0.09-11.02$ & 1.00 & $19.2^{\star}$ \\
\hline HLA-A*68:01 & 10 & 6 & 1.83 & $0.15-22.36$ & 0.53 & $1.92^{*}$ \\
\hline HLA-A*26:01 & 10 & 4 & 2.87 & $0.20-39.68$ & 0.43 & $1.92^{*}$ \\
\hline HLA-A*01:01 & 0 & 6 & 0.58 & $0.02-13.05$ & 1.00 & $11.5^{*}$ \\
\hline HLA-A*02:01 & 0 & 8 & 0.43 & $0.02-9.34$ & 1.00 & $5.77^{*}$ \\
\hline HLA-B*07:02 & 30 & 10 & 6.00 & $0.78-46.14$ & 0.10 & $3.84^{*}$ \\
\hline HLA-B*15:01 & 0 & 8 & 0.43 & $0.02-9.34$ & 1.00 & $1.92^{*}$ \\
\hline HLA-B*44:02 & 30 & 10 & 6.00 & $0.78-46.14$ & 0.10 & $1.92^{*}$ \\
\hline HLA-B*35:01 & 10 & 6 & 1.83 & $0.15-22.36$ & 0.53 & $7.69^{*}$ \\
\hline HLA-B*40:01 & 10 & 6 & 1.83 & $0.15-22.36$ & 0.53 & $0.96^{*}$ \\
\hline HLA-B*40:02 & 0 & 10 & 0.33 & $0.01-7.11$ & 0.55 & $1.10^{\dagger}$ \\
\hline HLA-B*40:06 & 0 & 4 & 0.85 & $0.03-20.45$ & 1.00 & $15.4^{\star}$ \\
\hline HLA-B*50:01 & 0 & 8 & 0.43 & $0.02-9.34$ & 1.00 & $1.92^{*}$ \\
\hline HLA-DRB1*15:01 & 10 & 22 & 0.27 & $0.02-2.77$ & 0.62 & $14.8^{*}$ \\
\hline HLA-DRB1*15:02 & 0 & 6 & 0.58 & $0.02-13.05$ & 1.00 & $7.4^{*}$ \\
\hline HLA-DRB1*07:01 & 30 & 20 & 2.66 & $0.37-19.06$ & 0.36 & $9.25^{*}$ \\
\hline HLA-DRB1*13:01 & 20 & 10 & 2.66 & $0.34-20.50$ & 0.56 & $1.85^{*}$ \\
\hline HLA-DRB1*10:01 & 10 & 8 & 1.31 & $0.11-15.03$ & 1.00 & $3.7^{*}$ \\
\hline HLA-DRB1*14:01 & 0 & 4 & 0.85 & $0.03-20.45$ & 1.00 & $0.92^{*}$ \\
\hline HLA-DRB1 *04:01 & 10 & 4 & 2.87 & $0.20-39.68$ & 0.43 & $0.92^{*}$ \\
\hline
\end{tabular}

MPE, maculopapular exanthem; HLA, human leukocyte antigen.

"Ref.8; 'Ref.9. 
92 Journal of Epilepsy Research Vol. 6, No. 2, 2016

Table 3. 4-digit HLA allele of all 33 subjects

\begin{tabular}{|c|c|c|c|c|c|c|}
\hline & HLA-A (Allele 1) & HLA-A (Allele 2) & HLA-B (Allele 1) & HLA-B (Allele 2) & HLA-DRB1 (Allele & 1) HLA-DRB1 (Allele 2) \\
\hline Case 1 & 33:01 & 68:01 & $35: 03$ & 40:01 & 13:01 & $14: 04$ \\
\hline Case 2 & 03:02 & $26: 01$ & 07:02 & 07:02 & 07:01 & 10:01 \\
\hline Case 3 & $24: 02$ & 33:01 & 07:02 & 44:02 & 13:01 & $15: 01$ \\
\hline Case 4 & 02:03 & $02: 11$ & $15: 02$ & 40:06 & 15:01 & $15: 02$ \\
\hline Case 5 & $11: 01$ & $68: 01$ & 07:02 & $15: 01$ & $15: 01$ & $15: 01$ \\
\hline Case 6 & $11: 01$ & $68: 01$ & $13: 16$ & $52: 01$ & 04:01 & $14: 01$ \\
\hline Case 7 & 11:01 & 24:01 & 44:02 & 44:02 & 07:01 & 07:01 \\
\hline Case 8 & $32: 01$ & $33: 03$ & $18: 01$ & $35: 01$ & 04:01 & $11: 01$ \\
\hline Ctrl 1 & 01:01 & $24: 01$ & $35: 01$ & $52: 01$ & $12: 02$ & $15: 02$ \\
\hline Ctrl 2 & 11:01 & $11: 01$ & $35: 03$ & 44:02 & $13: 15$ & $14: 19$ \\
\hline Ctrl 3 & $02: 11$ & $11: 01$ & $40: 02$ & $41: 01$ & $11: 01$ & $14: 01$ \\
\hline Ctrl 4 & 02:05 & $11: 01$ & 07:02 & 44:02 & $15: 01$ & $15: 02$ \\
\hline Ctrl 5 & $26: 01$ & $31: 01$ & $44: 02$ & $51: 01$ & $15: 01$ & $15: 01$ \\
\hline Ctrl 6 & 02:01 & 33:01 & $13: 01$ & $15: 01$ & 04:06 & 13:01 \\
\hline Ctrl 7 & 11:01 & 24:02 & $18: 01$ & $52: 01$ & $15: 01$ & $15: 01$ \\
\hline Ctrl 8 & 11:01 & 11:01 & 15:01 & $40: 02$ & 04:08 & 07:08 \\
\hline Ctrl 9 & 11:01 & 24:02 & 15:01 & 40:06 & 10:01 & $12: 01$ \\
\hline Ctrl 10 & $24: 02$ & 24:07 & $15: 02$ & 40:06 & $15: 01$ & $15: 02$ \\
\hline Ctrl 11 & $32: 01$ & $33: 03$ & 07:02 & $40: 02$ & 13:01 & 15:01 \\
\hline Ctrl 12 & 11:01 & 24:02 & 15:01 & 40:02 & 10:01 & $12: 01$ \\
\hline Ctrl 13 & 11:01 & $68: 01$ & 07:02 & 40:02 & 04:01 & 07:01 \\
\hline Ctrl 14 & $24: 02$ & $26: 01$ & 07:02 & $44: 02$ & 07:01 & $15: 01$ \\
\hline Ctrl 15 & 03:01 & 03:01 & $50: 01$ & $57: 01$ & 03:01 & 07:01 \\
\hline Ctrl 16 & 02:01 & $11: 01$ & 13:01 & $50: 01$ & $10: 01$ & 13:01 \\
\hline Ctrl 17 & $24: 01$ & $68: 01$ & 07:02 & $50: 01$ & 07:01 & 07:01 \\
\hline Ctrl 18 & 03:01 & 24:01 & 35:01 & 40:01 & 07:01 & 11:01 \\
\hline Ctrl 19 & 03:01 & $33: 03$ & $35: 01$ & 44:02 & 04:01 & 07:01 \\
\hline Ctrl 20 & $30: 01$ & $68: 01$ & $51: 01$ & 53:01 & 13:01 & $14: 01$ \\
\hline Ctrl 21 & 01:01 & 02:01 & $37: 01$ & 53:01 & 10:01 & 13:01 \\
\hline Ctrl 22 & 01:01 & 24:06 & $52: 01$ & $57: 01$ & 03:01 & 07:01 \\
\hline Ctrl 23 & 11:01 & $33: 03$ & 40:01 & $52: 01$ & $14: 07$ & $15: 01$ \\
\hline Ctrl 24 & 02:01 & 03:01 & 40:01 & $52: 01$ & 07:01 & 15:01 \\
\hline Ctrl 25 & 01:02 & 11:01 & 50:01 & 57:01 & 03:01 & 15:01 \\
\hline
\end{tabular}

HLA, human leukocyte antigen.

Table 4. Case series and reports of cutaneous adverse effects related to LEV

\begin{tabular}{lllc}
\hline Serial number & \multicolumn{1}{c}{ Case detail } & Type of cADR & Reference number \\
\hline 1 & Neonate with perinatal asphyxia, pneumothorax & $\begin{array}{c}\text { Erythematous rash and anaphylactic } \\
\text { reaction } \\
\text { Angioedema }\end{array}$ & 10 \\
2 & 33 year old lady with drug-refractory epilepsy & DRESS & 11 \\
3 & 55 year old man with intracranial haemorrhage & TEN & 12 \\
4 & 2 cases of 20 and 29 year old women with epilepsy & 4 \\
5 & 2.25 year old female after surgery for congenital heart disease and SJS & 2 \\
6 & cardiorespiratory arrest & DRESS & 3 \\
7 & 18 year old man & Morbilliform rash & 13 \\
8 & 64 year old man with basal ganglia mass & Hair loss & 14 \\
9 & 5 Cases & MPE, Morbilliform rash & 15 \\
\hline
\end{tabular}

LEV, levetiracetam; CADR, cutaneous adverse drug reaction; SJS, Stevens Johnson syndrome; DRESS, drug reaction eosinophilia and systemic symptoms; TEN, toxic epidermal necrolysis; MPE, maculopapular exanthem. 


\section{Discussion}

Cutaneous drug reactions due to LEV are rare, and only case reports and small series are published (Table 4). ${ }^{2-4,10-15}$ In a systematic review of ten randomised controlled trials by Mbizvo et al, adverse effect profile of LEV in patients of drug-resistant epilepsy was studied, and rash due to LEV was reported in only two studies. It was noted that only symptoms affecting more than 5 or $10 \%$ of participants of the groups were published in these ten trials. ${ }^{16}$ In the study by Cheung et al, out of 55 patients of CADR due to various AEDs identified over 16.5 years, there was only one patient with rashes due to $\mathrm{LEV}$, though one of the reasons maybe that being a newly introduced AED it may not have been prescribed much in the initial few years. ${ }^{17}$ Out of 589 patients screened in our study over two years, there were only eight cases of CADRs - this explains the small sample-size of cases. Two of the patients with skin reactions had SJS - though severe cutaneous drug reactions like SJS/TEN and DRESS are much less common than MPE. Both our SJS patients were on LEV monotherapy at onset of the symptoms therefore likelihood of the reaction being LEV-induced is high; most case-reports of these life-threatening CADRs with LEV are of patients on other drugs also, including AEDs.

Association of HLA alleles has been studied mainly in aromatic $A E D s$, as they are the cause of most CADRs, in particular CBZ, and certain HLA alleles predispose patients of some ethnicities to specific CADRs due to AEDs. In the European and the Chinese populations, presence of the allele HLA-A*31:01 appears to predispose to CBZ-induced DRESS, as found in an international study and meta-analysis. ${ }^{18}$ HLA $B^{*} 15: 02$ is associated with SJS and TEN due to CBZ intake in Han Chinese population, but not with MPE. ${ }^{6}$ Evidence from another meta-analysis of nine case-control studies by Li $X$ et al shows significant association between HLA-A* 15:02 and PHT and LTG-induced SJS/TEN.$^{19} A$ pilot-study showed that the same allele may be associated with OXC-induced mild cutaneous reactions. ${ }^{20}$ It was shown in a prospective study of 252 patients, 5 developed CADRs to OXC. Out of these 5 patients, 1 was positive for HLA-B*15:02. ${ }^{21}$ In our study we have noted seen that HLA-A*33:01, though not statistically significant, is present in cases of MPE compared to controls $(\mathrm{OR}=6)$. The $p$-values were not significant (0.31), when differences in AF or carrier rates were considered for calculation, and the confidence intervals may have been wide, due to the small sample size of cases.

Studies have shown HLA-B* 15:02 to be associated with SJS in Indians as well. Mehta et al found 6 out of 8 CBZ-induced SJS patients from the western state of Gujarat HLA B* 15:02 positive, but none among 10 healthy controls. Other studies were on North and South Indian origin patients in which also a significant association was found between the presence of this allele and SJS due to CBZ. ${ }^{22,23}$ However, one of our SJS patients was positive for HLA-B* 15:02. Interestingly, she had been on CBZ for over a year without any skin or mucosal reactions, and recovered as soon as her LEV was stopped. Since she had tested positive for this allele, she was shifted to Valproate from CBZ. None of the MPE cases came out to be positive for 15:02. The fact that India has a racially diverse population may be an explanation. Also there are probably other factors, like interaction of the HLA molecule with specific T-cell receptors also contributing to a CADR. HLA-A*31:01 was shown to be linked to all types of cutaneous reactions (MPE, DRESS and SJS) in the European population in a genome-wide association based study and Hu et al had four OXC-induced MPE cases positive for HLA-B*15:02 out of nine, significant when compared with population controls. ${ }^{7,20} \mathrm{~A}$ significant association of HLA-A*24:02 with LTG induced MPE was found in study of 21 Korean patients. ${ }^{24}$

Possible protective effects of the presence of HLA-B* $40: 01$ and HLA-B*58:01 have also been demonstrated on the basis of significantly higher rates of occurrence among AED-tolerant controls. ${ }^{17}$ We found HLA-A*11:01 positivity in 11 out of the 25 LEV-tolerant controls as against 3 out of eight cases, the AF being $26 \%$ and 10\% respectively; the AF among healthy controls from the same North Indian states was $12.5 \%$, which may suggest that the presence of this allele may be protective, though not statistically significant. ${ }^{8} \mathrm{~A}$ limitation of our study is that we did not simultaneously recruit healthy population controls. However, we have quoted published AF values from the same population for the alleles found in our cases and controls (Table 2). These studies, by Rajalingam et al and Rani et al had HLA-typing of 52 and 90 healthy North-Indian subjects respectively.

Another very recent study in the Chinese population, with nine cases of mild rashes due to LEV has also shown no significant association between HLA alleles and LEV-induced cutaneous rashes. ${ }^{25}$

Cutaneous reactions to LEV, though quite unusual, have a wide spectrum of phenotypes including severe reactions like SJS/TEN and DRESS. HLA-A*33:01 was higher among MPE cases as compared to tolerant controls whereas HLA-A*11:01 was higher in tolerant controls than MPE cases, although neither association was statistically significant. We did not find any significant association between HLA alleles and LEV induced cutaneous drug rashes but a larger sample-size of cases in a multicentre study may be required in order to confirm the findings. 


\section{Acknowledgement}

This work was supported by Department of Biotechnology (DBT), Ministry of Science and Technology, Government of India. Project code: BT/PR3436/MED/30/651/2011.

\section{Conflict of Interest}

None of the authors has any conflict of interest to disclose.

\section{References}

1. Sharma VK, Sethuraman G, Kumar B. Cutaneous adverse drug reactions: clinical pattern and causative agents--a 6 year series from Chandigarh, India. J Postgrad Med 2001;47:95-9.

2. Zou LP, Ding CH, Song ZJ, Li XF. Stevens-Johnson syndrome induced by levetiracetam. Seizure 2012;21:823-5.

3. Hall DJ, Fromm JS. Drug reaction with eosinophilia and systemic syndrome in a patient taking phenytoin and levetiracetam: a case report. J Med Case Rep 2013;7:2.

4. Duong TA, Haddad C, Valeyrie-Allanore L, Sbidian E, Chosidow O, Wolkenstein P. Levetiracetam: a possible new inducer of toxic epidermal necrolysis and Stevens-Johnson syndrome in 2 cases. JAMA Dermatol 2013;149:113-5.

5. FDA Approved Labeling text dated 16 Dec 2011. Reference ID: 3060029, www.fda.gov/downloads/Drugs/DrugSafety/UCM152832.pdf.

6. Chung WH, Hung SI, Hong HS, et al. Medical genetics: a marker for Stevens-Johnson syndrome. Nature 2004;428:486.

7. McCormack M, Alfirevic A, Bourgeois S, et al. HLA-A 3101 and carbamazepine-induced hypersensitivity reactions in Europeans. $N$ Eng/ J Med 2011;364:1134-43.

8. Rajalingam $R$, Krausa P, Shilling HG, et al. Distinctive KIR and HLA diversity in a panel of north Indian Hindus. Immunogenetics 2002;53:1009-19.

9. Rani $R$, Marcos $C$, Lazaro AM, Zhang $Y$, Stastny P. Molecular diversity of HLA-A, $-B$ and $-C$ alleles in a North Indian population as determined by PCR-SSOP. Int I Immunogenet 2007;34:201-8.

10. Koklu E, Ariguloglu EA, Koklu S. Levetiracetam-Induced anaphylaxis in a neonate. Pediatr Neurol 2014;50:192-4.

11. Alkhotani A, McLachlan RS. Levetiracetam induced angioedema in a patient with previous anticonvulsant hypersensitivity reaction to phe- nytoin and lamotrigine. Seizure 2012;21:407-8.

12. Eleni K. Dress syndrome induced by levetiracetam. J Eur Acad Dermatol Venereol 2015;29:377-8.

13. Jones RT, Evans W, Mersfelder TL, Kavanaugh K. Rare red rashes: a case report of levetiracetam-Induced cutaneous reaction and review of the literature. Am J Ther J 2016;23:e944-6.

14. Zou X, Hong Z, Zhou D. Hair loss with levetiracetam in five patients with epilepsy. Seizure 2014;23:158-60.

15. Bhoi Sk, Kalita J, Misra Uk. Skin rash following levetiracetam. Seizure 2016;37:45-7.

16. Mbizvo GK, Dixon P, Hutton JL, Marson AG. The adverse effect profile of levetiracetam in epilepsy: a more detailed look. Int I Neurosci 2014; 124:627-34.

17. Cheung YK, Cheng SH, Chan EJ, Lo SV, Ng MH, Kwan P. HLA-B alleles associated with severe cutaneous reactions to antiepileptic drugs in Han Chinese. Epilepsia 2013;54:1307-14.

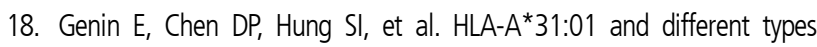
of carbamazepine-induced severe cutaneous adverse reactions: an international study and meta-analysis. Pharmacogenomics / 2014; 14:281-8.

19. Li $X$, Yu K, Mei $S$, et al. HLA-B*15:02 increases the risk of phenytoin or lamotrigine induced Stevens-Johnson Syndrome/toxic epidermal necrolysis: evidence from a meta-analysis of nine case-control studies. Drug Res (Stuttg) 2015;65:107-11.

20. Hu FY, Wu XT, An DM, Yan B, Stefan H, Zhou D. Pilot-association study of oxcarbazepine-induced mild cutaneous reaction with HLA B*1502 allele in Chinese Han population. Seizure 2011;20:160-2.

21. He N, Min FL, Shi YW, et al. Cutaneous reactions induced by oxcarbazepine in Southern Han Chinese: incidence, features, risk factors and relation to HLA-B alleles. Seizure 2012;21:614-8.

22. Aggarwal R, Sharma M, Modi M, Garg VK, Salaria M. HLA-B* 1502 is associated with carbamazepine induced Stevens-Johnson syndrome in North Indian population. Human Immunol 2014;75:1120-2.

23. Khor AH, Lim KS, Tan CT, Wong SM, Ng CC. HLA-B*15:02 association with carbamazepine-induced Stevens-Johnson syndrome and toxic epidermal necrolysis in an Indian population: a pooled-data analysis and meta-analysis. Epilepsia 2014;55:e120-4.

24. Wang $X Q$, Lv $B$, Wang HF, et al. Lamotrigine induced DIHS/DRESS: Manifestations, treatment, and outcome in 57 patients. Clin Neurol Neurosurg 2015;138:1-7.

25. Hu FY, Wang W, Ren JC, An DM, Chen JN, Zhou D. Levetiracetam-induced cutaneous adverse drug reactions were not associated with HLA genes in a small sample of Chinese patients with epilepsy. Epilepsy Res 2016;124:12-5. 